\title{
User Interface Control Framework of Sexual Violence Prevention Education
}

\author{
Yunsick Sung ${ }^{1}$, Donguk Kim ${ }^{1}$, Hyung Jin Park ${ }^{2}$ and Kyung Min Park ${ }^{3 *}$ \\ ${ }^{1}$ Faculty of Computer Engineering, Keimyung University, Daegu 42601, South \\ Korea, \\ ${ }^{2}$ College of Fine Art, Keimyung University, Daegu 42601, South Korea, \\ ${ }^{3}$ College of Nursing, Keimyung University, Daegu 42601, South Korea, \\ \{yunsick,donguk, phj, kmp\}@kmu.ac.kr \\ *kmp@kmu.ac.kr
}

\begin{abstract}
This paper proposes a user interface control framework of sexual violence prevention education Apps. To conduct the control of the properties of UI elements such as colors and sizes, the frequency, the utilization, the relationship of UI elements are considered. In the experiment, the application, "Sexual violence! How should I do?", was utilized applying the proposed framework. The sizes of the buttons of the application was resized based on the data of the utilization of four subjects.
\end{abstract}

Keywords: Human-computer interaction, User interface, Sexual violence, Bayesian probability.

\section{Introduction}

Recently sex violence-related problems are emerged diversely as a serious issue such as sexual harassments, sexual abuse, sexual assault, stalking, and cyber sexual violence [1-5] and are increased [6]. Therefore, the importance of the sexual protection educations for primary school student is also increased. Tradition sexual protection educations are based on books, CDs and animations. However, new education paradigm that utilizes PCbased applications and mobile phone-based apps is issued. The applications and apps has the advantages that students can learn those contents based on the interaction between those contents and the students.

The UI control framework by utilizing Bayesian probability was introduced [7]. By considering the frequency and the importance of UI elements, the size of UI elements are adjusted. By changing the sizes of all UI elements, the UI elements of more important scenarios relatively are performed. However, diverse kinds of factors need to be considered more as follow. First, the importance of the UI elements of performed scenarios is low comparing to that of not performed scenarios. Next, related scenarios requires to be performed to be compared each other. Therefore, right after one of related scenarios is played, one of the UI elements of the scenarios that are related and are not performed yet. Next, the importance of the UI elements can be increased or decreased depending on the time or one the process of education processes. As results, new novel UI control framework is required to improve the effects of PC-based and mobile phone-based sexual violence protection education.

This paper proposes a user interface control framework of sexual violence protection education for elementary school students. Given that bigger UIs induces user to utilize own UIs, more effective scenarios are played by the elementary school students by making the sizes of UIs bigger.

* Corresponding Author 
This paper is consisted as follow. Section 2 proposes an UI control framework. Section 3 shows the result of UIs by applying the proposed UI control framework. Finally Section 4 concludes.

\section{UI Control Framework for Dynamic User Interfaces}

This paper introduces an improved UI control framework that is utilized for improving the learning effect of sexual violence protection education applications and apps. In the previous research [7], the Thd $i$ th UI probability $p_{i}$ is defined as $p_{i}=\frac{c_{i}}{\sum_{j=1}^{n} c_{j}}$ based on Bayesian probability [8] of the $i$ th UI element according to the consideration of the frequency of utilized UI elements where $n$ is the number of UI elements and the UI count $c_{i}$ is the number of the utilization of the $i$ th UI elements. Bayesian probability is utilized diversely to improve UIs [9-10]. Then each UI value $v_{i}$ of elements is calculated by the equation of $v_{i}=p_{i} \times w_{i, 1} \times w_{i, 2} \times \ldots \times w_{i, m}$ where $m$ is the number of weights and the weight $w_{i, m}$ is the $m$ th weight of the $i$ th UI element. The $i$ th UI element is changed considering the UI value $v_{i}$ depending on contents.

The UI control framework in this paper contains the improved factors as follow. First, the $i$ th UI probability $p_{i}$ is considered as Equation (1). Given that the UI probability $p_{i}$ denotes how much users utilize, the UI probability $p_{i}$ is the key in this framework.

$$
v_{i}=p_{i(1)}
$$

Next, the importance of the UI elements is reflected to consider the importance of the UI elements of not performed scenarios as shown in Equation (1).

$$
v_{i}=w_{p} \times p_{i}+w_{e} \times e_{i(1)}
$$

where the utilization factor $e_{i}$ is the $i$ th execution importance factor. The amounts how much the UI probability $p_{i}$ and the utilization factor $e_{i}$ are affected are adjusted by weights, $w_{p}$ and $w_{e}$. The utilization factor $e_{i}$ is defined as Equation (2).

$$
e_{i}=1-u_{i(2)}
$$

where the usage $u_{i}$ is initialized by 0 and is set by 1 when the $i$ th UI element is utilized. Therefore, if the $i$ th UI element is utilized, the UI value $v_{i}$ is changed to 0 .

Next, the relationship among UI elements is reflected to perform the related scenarios that require to be performed in order. The relationships among UI elements are expressed as UI relationship table in advance, and then applied as Equation (3).

$$
\left\{\begin{array}{l}
\text { if } t=1 \text { then } v_{i, t}=w_{p} \times p_{i}+w_{e} \times e_{i, t} \\
\text { else } v_{i, t}=\left(w_{p} \times p_{i}+w_{e} \times e_{i, t}\right) \times r_{i, t-1(3)}
\end{array}\right.
$$

where the UI relation $r_{i, t-1}$ is the relationship between the $i$ th UI element and the performed UI element at time $\mathrm{t}-1$. The utilization factor $e_{i, t}$ is the $e_{i}$ at time $t$. When time $t$ is 1 , because of no performed UI elements, the UI relation $r_{i, t-1}$ cannot be utilized.

Finally, a specific weight is required depending on education applications and apps. Therefore, an additional weight is added as Equation (5).

$$
\left\{\begin{array}{l}
\text { if } t=1 \text { then } v_{i, t}=w_{p} \times p_{i}+w_{e} \times e_{i, t}+w_{i} \\
\text { else } v_{i, t}=\left(w_{p} \times p_{i}+w_{e} \times e_{i, t}\right) \times r_{i, t-1}+w_{i(5)}
\end{array}\right.
$$

The user interface control framework in this paper is consisted as shown in Figure 1 to calculate UI values of UI elements. The framework has six modules, which are divided into two groups: One group contains UI counter, UI probability calculator and UI relationship applier and the other group contains utilization factor decider and mixer 


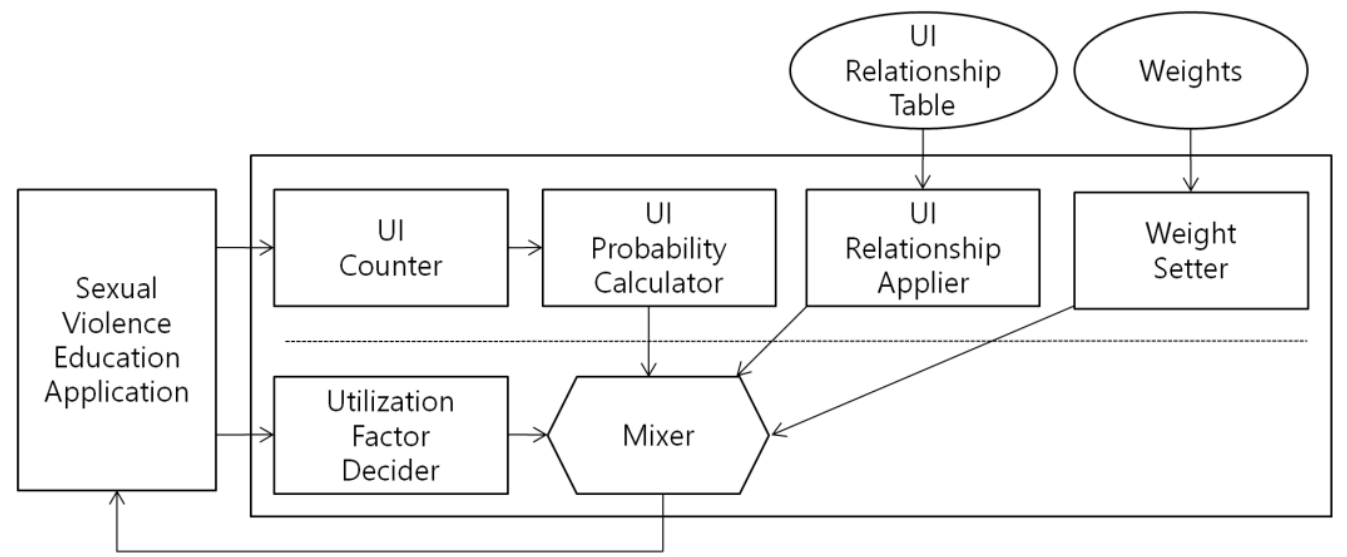

\section{Figure 1. User Interface Control Framework. The Framework Contains UI Counter, UI Probability Calculator, UI Relationship Applier, Utilization Factor Decider, Weight Setter, And Mixer To Control The Properties Of Uis}

The prior group is for calculating UI properties and defining all parameter of the framework. Therefore, all modules in the prior group are performed before executing education applications and apps. However, the modules in posterior group are performed repeatedly to check and apply whether each UI element is utilized or not during executing education application and apps.

UI counter analyzes the utilization of UI elements of sexual violence prevention education applications and apps and then counts the number of the utilization. UI Probability calculator calculates the UI probabilities based on the count numbers. UI relationship applier and weight setter receives UI relationship table and weights, and then transfers to mixer. Utilization factor decider treats the utilization of UI elements. Mixer receives all values from UI probability calculator, UI relationship applier, weight setter, and utilization factor decider.

\section{Experiment}

In the experiment, the application, "Sexual violence! How should I do?", was utilized after applying the proposed framework. The application is consisted of three stages as shown in Figure 2: Title stage, map stage, and scenario stage. During the scenario stage, play mode, question mode, and result mode are performed.

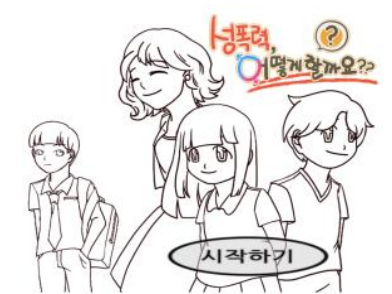

(a) Title Stage

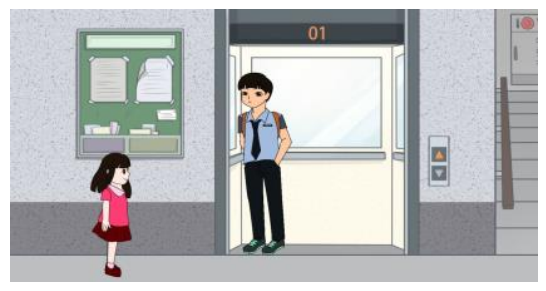

(c) Play Mode Of Scenario Stage

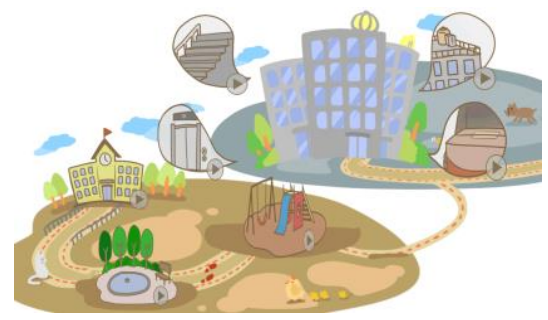

(b)

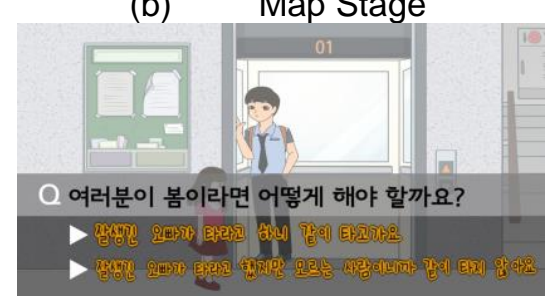

(d) Question Mode Of Scenario Stage 


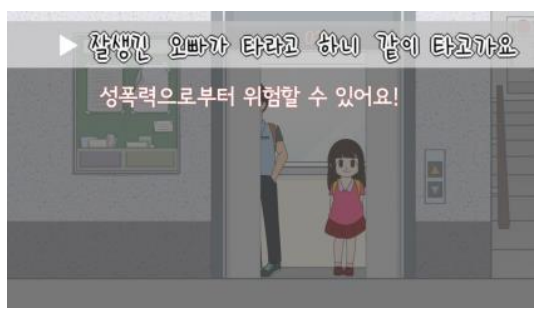

(e) Result Mode of Scenario Stage

\section{Figure 2. Three Stages Of "Sexual Violence! How Should I Do?": Title Stage, Map Stage And Scenario Stage. One Of Scenarios Is Selected On Map Stage And Is Played Through Play Mode, Question Mode, Result Mode}

Map stage provides the UI as buttons in a map. When users select one of scenarios by clicking the corresponding buttons, the scenario is played during scenario stage. The processes of scenario stage are divided into three modes: Play mode, question mode, and result mode. During play mode, users watch the situations when sexual violence can be occurred. After finishing playing a single scenario, the corresponding question is displayed and users should choose one of answers on the bottom list. The result of the chosen answer is evaluated and displayed during result mode. When wrong answers are selected, the guide line how to act is introduced.

The application contains seven scenarios as shown in Table 1. The scenarios are made according to the interview with assistants in a sexual violence counseling center and with teachers in elementary schools. The application is for elementary school students. Therefore, sexual violence places are selected considering the elementary school students.

Table 1. The Scenarios Of Sexual Violence Protection Education Application

\begin{tabular}{|c|c|}
\hline Playground & First seen brother says "Hang out together" and touches my body. \\
\hline School & My friend shows me a nude picture in his mobile phone. \\
\hline Park & $\begin{array}{l}\text { While I am in woman toilet, an unknown uncle enters the woman } \\
\text { toilet. }\end{array}$ \\
\hline Delivery & Deliverer comes at home when I stay alone. \\
\hline Elevator & Unknown person says "Ride an elevator." \\
\hline Rooftop & Relative brother kisses me when we hang out together on rooftop. \\
\hline Stairs & $\begin{array}{l}\text { Grandfather in neighbor asks me to help him to transfer his luggage } \\
\text { and touches my body. }\end{array}$ \\
\hline
\end{tabular}

There are seven buttons as UI elements in Figure 2(b) where each button is mapped to a single corresponding scenario. In the experiment, four subjects were involved. Table 2 shows the result of the selected orders of scenarios per each subject. We assume that subjects do not perform each scenario more than one time. 
Table 2. The Selected Scenarios in Order Depending on Four Subjects

\begin{tabular}{|c|c|c|c|c|c|c|c|}
\hline $\begin{array}{c}\text { Subject } \\
1\end{array}$ & $\begin{array}{c}\text { Schoo } \\
1\end{array}$ & Park & $\begin{array}{c}\text { Play- } \\
\text { ground }\end{array}$ & $\begin{array}{c}\text { Deliv } \\
\text { ery }\end{array}$ & $\begin{array}{c}\text { Elevat } \\
\text { or }\end{array}$ & $\begin{array}{c}\text { Rooft } \\
\text { op }\end{array}$ & Stairs \\
\hline $\begin{array}{c}\text { Subject } \\
2\end{array}$ & $\begin{array}{c}\text { Schoo } \\
1\end{array}$ & Park & $\begin{array}{c}\text { Play- } \\
\text { ground }\end{array}$ & $\begin{array}{c}\text { Elevat } \\
\text { or }\end{array}$ & Stairs & $\begin{array}{c}\text { Rooft } \\
\text { op }\end{array}$ & $\begin{array}{c}\text { Deliv } \\
\text { ery }\end{array}$ \\
\hline $\begin{array}{c}\text { Subject } \\
3\end{array}$ & $\begin{array}{c}\text { Elevat } \\
\text { or }\end{array}$ & $\begin{array}{c}\text { Play- } \\
\text { ground }\end{array}$ & $\begin{array}{c}\text { Deliv } \\
\text { ery }\end{array}$ & Park & Stairs & $\begin{array}{c}\text { Schoo } \\
1\end{array}$ & $\begin{array}{c}\text { Rooft } \\
\text { op }\end{array}$ \\
\hline $\begin{array}{c}\text { Subject } \\
4\end{array}$ & Park & $\begin{array}{c}\text { Rooft } \\
\text { op }\end{array}$ & $\begin{array}{c}\text { Play- } \\
\text { ground }\end{array}$ & $\begin{array}{c}\text { Deliv } \\
\text { ery }\end{array}$ & Stairs & $\begin{array}{c}\text { Elevat } \\
\text { or }\end{array}$ & $\begin{array}{c}\text { Schoo } \\
1\end{array}$ \\
\hline
\end{tabular}

To reveal the favorite UI elements, the top 4 selections per each subject are only considered to calculate the UI probabilities. Table 3 shows the selected count of the UI buttons.

Table 3. The Count of the UI Buttons of Scenarios

\begin{tabular}{|l|c|c|c|c|c|c|c|}
\hline & Playground & School & Park & Delivery & Elevator & Rooftop & Stairs \\
\hline Play & 4 & 2 & 4 & 3 & 2 & 1 & 0 \\
\hline
\end{tabular}

Table 4 shows the result the UI probabilities of the UI buttons based on the count of the UI buttons. The UI probabilities of playground and park were highest.

Table 4. The UI Probabilities of the UI Buttons

\begin{tabular}{|c|c|c|c|c|c|c|c|}
\hline & Playground & School & Park & Delivery & Elevator & Rooftop & Stairs \\
\hline$p_{i}$ & $\mathbf{0 . 2 5}$ & 0.125 & $\mathbf{0 . 2 5}$ & 0.1875 & 0.125 & 0.0625 & 0 \\
\hline
\end{tabular}

In the experiment, the UI relationship table is defined as shown in Table 6. For example, the relationship of playground after school scenario is defined by 2 .

Table 5. The UI Relationship Table

\begin{tabular}{|c|c|c|c|c|c|c|c|}
\hline & $\begin{array}{c}\text { Playgro } \\
\text { und }\end{array}$ & $\begin{array}{c}\text { Scho } \\
\text { ol }\end{array}$ & Park & $\begin{array}{c}\text { Deliv } \\
\text { ery }\end{array}$ & $\begin{array}{c}\text { Eleva } \\
\text { tor }\end{array}$ & $\begin{array}{c}\text { Rooft } \\
\text { op }\end{array}$ & $\begin{array}{c}\text { Stair } \\
\text { s }\end{array}$ \\
\hline $\begin{array}{c}\text { Playgro } \\
\text { und }\end{array}$ & & 1 & 1 & 1 & 2 & 1 & 2 \\
\hline School & 2 & 1 & 1 & 2 & 1 & 2 \\
\hline Park & 1 & 1 & 1 & 1 & 2 & 1 & 1 \\
\hline Delivery & 1 & 1 & 1 & 1 & 1 & 1 \\
\hline Elevator & 1 & 1 & 1 & 2 & 2 & 1 \\
\hline Rooftop & 1 & 1 & 1 & 1 & 1 & 1 \\
\hline Stairs & 1 & & & & & 1 & 1 \\
\hline
\end{tabular}


The weights $w_{p}$ and $w_{e}$ are set through experiments by 0.4 and 0.6 . Then the subweights of the weight $w_{i}$ is defined as shown in Table 6. the weight $w_{i, 1}$ is the $i$ th weight considering where sexual violence are invoked by whom people know and the $i$ th weight $w_{i, 2}$ is defined considering where children sexual violence are happen frequently. In the experiment, the weight $w_{i}$ is defined by adding the weight $w_{i, 1}$ to the weight $w_{i, 2}$.

Table 6. The Sub-Weights For The Weight $W_{i}$

\begin{tabular}{|c|c|c|c|c|c|c|c|}
\hline & $\begin{array}{c}\text { Playgroun } \\
\mathrm{d}\end{array}$ & $\begin{array}{c}\text { Schoo } \\
1\end{array}$ & Park & $\begin{array}{c}\text { Delive } \\
\text { ry }\end{array}$ & $\begin{array}{c}\text { Elevat } \\
\text { or }\end{array}$ & $\begin{array}{c}\text { Rooft } \\
\text { op }\end{array}$ & Stairs \\
\hline$w_{i,}$ & & 0.25 & & & & 0.25 & 0.25 \\
\hline$w_{i,}$ & 0.5 & & & 0.25 & & & 0.5 \\
\hline
\end{tabular}

Until the calculation of the weight, there were four subjects. After analyzing the utilization of the UI buttons, all parameters are set. Then a new subject was involved to evaluate the set parameters. Table 7 shows the invoked time of each scenarios of the new subject after applying all parameters to the application.

Table 7. The Utilization Factors

\begin{tabular}{|c|c|}
\hline$e_{i}$ & Invoked time (s) \\
\hline Playground & 2.873912 \\
\hline School & 5.077587 \\
\hline Park & 8.714642 \\
\hline Delivery & 11.73481 \\
\hline Elevator & 14.29029 \\
\hline Rooftop & 15.77433 \\
\hline Stairs & 16.70768 \\
\hline
\end{tabular}

Whenever the new subject selected one of the UI buttons, the corresponding utilization factor was recalculated as shown in Figure 3. When all UI buttons were selected, each UI value became the same value with the product of the UI probability $p_{i}$ and the weight $w_{p}$. As results, the UI values were changed based on the changed utilization factors as shown in Figure 4. 


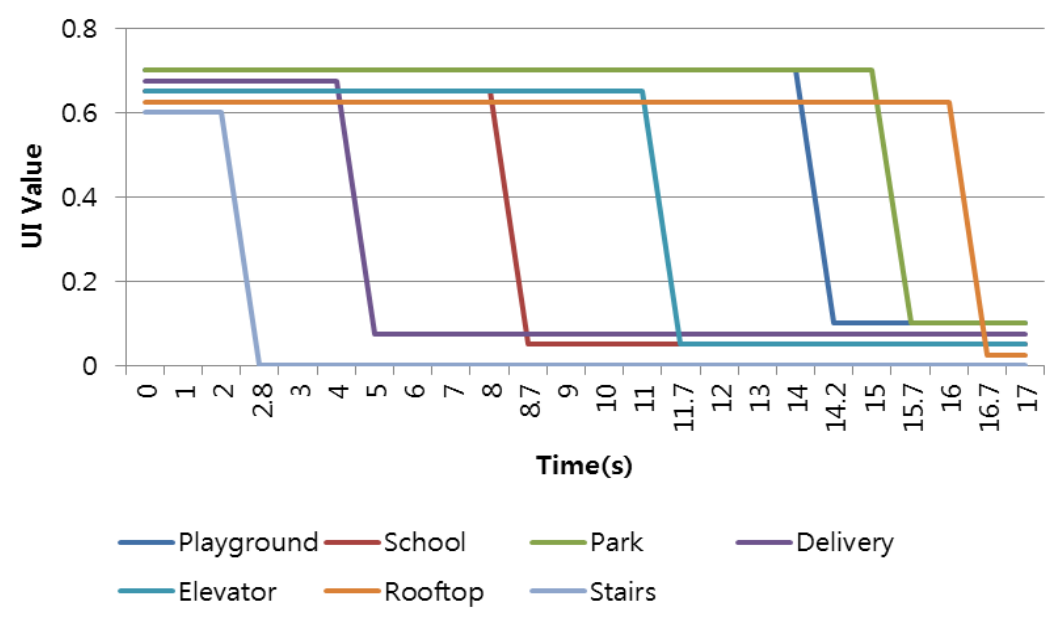

Figure 3. The Changes of the Sum of the Utilization Factors and the UI Probability

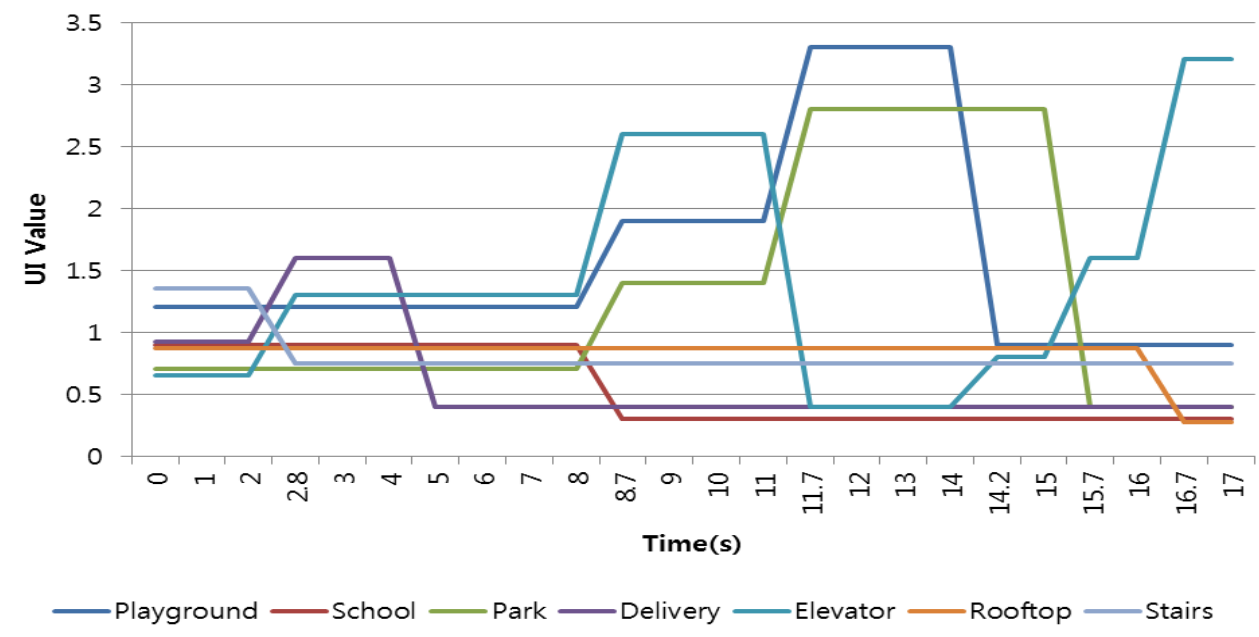

Figure 4. The Changes of the UI Values

Figure 5 shows the final ranks of the UI buttons during the new subject played all scenarios. Figure 6 shows the screenshot of the map stage while the new subject utilizes the application.

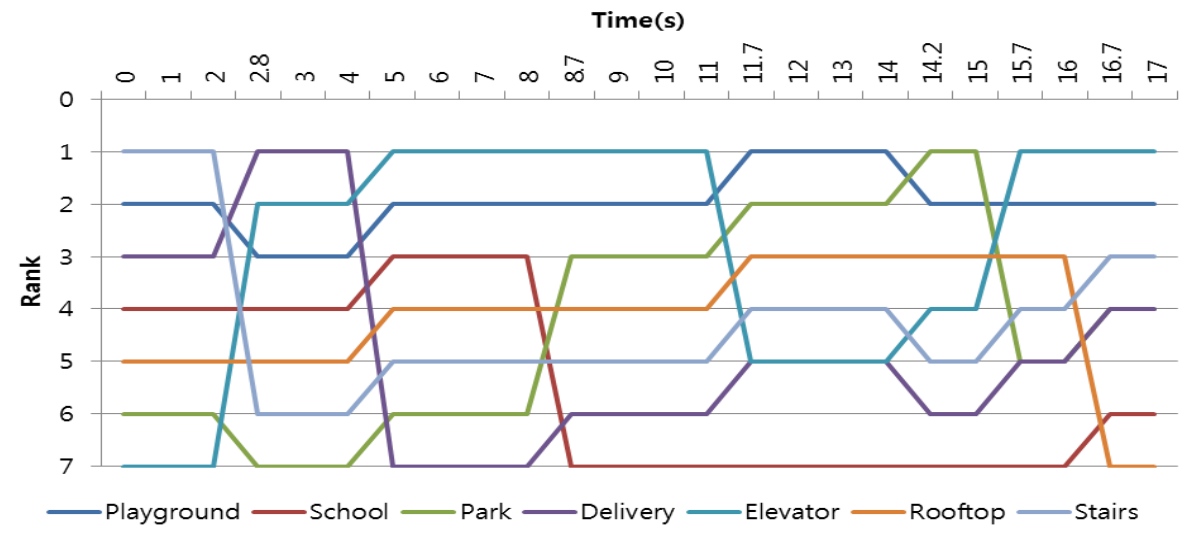

Figure 5. Final Rank 

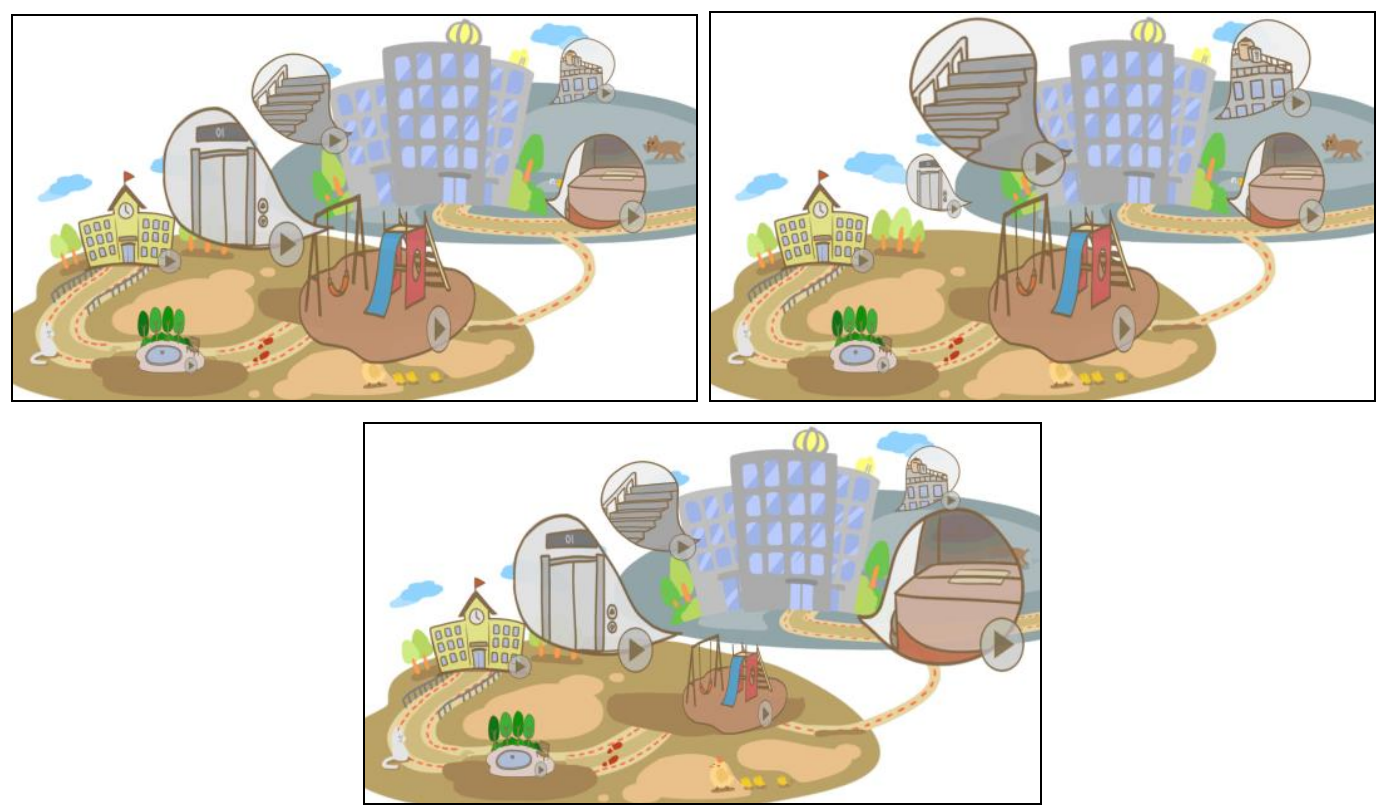

\section{Figure 6. Screenshot of the UI Buttons of the Sexual Violence Protection Education Application}

\section{Conclusion}

This paper proposed a UI control framework for sexual violence prevention education apps. The frequency of the important UI elements can be increased by changing the size of UI elements. The importance of UI elements can be adjusted by controlling the weights.

In the experiment, the proposed method was applied to the application, "Sexual violence! How should I do?". Four subjects were involved to set the parameters of the proposed method and then on additional subject participated in the experiment to validate the proposed method with the parameters. Depending on the plays of scenarios, the sizes of the buttons in the map of the application were adjusted. We confirm that users of the application can be induced by the change the shape of UI elements with the proposed medthod.

\section{Acknowledgment}

This research was supported by Basic Science Research Program through the National Research Foundation of Korea(NRF) funded by the Ministry of Education(NRF2015009659)

\section{References}

[1] K. Lee and M. Do, "The Effectiveness of the Child Sexual Violence Prevention Coaching Programs for Children into the Childrenl's Sexual Knowledge and the ability of Coping with Sexual Matters and Violences", Korean Journal of Parent Education Research, vol. 8, no. 1, (2011), pp. 45-67.

[2] J.A. Carter and E.A. Frankel, "The Effect of Teacher Training Program in Family Life and Human Sexuality on the Knowledge and Attitude of Public School Teachers", Journal of School Health, vol. 53, no. 4, (1983), pp. 459-462.

[3] D. Kirby, "Effective Approaches to Reducing Adolescent Unprotected Sex, Pregnancy, and Childbearing. Journal of Sex Research, vol. 39, no. 1, (2002), pp. 51-57.

[4] G.S. Parcel and D. Luttmann, "Evaluation of Sex Education Course for Young Adolescents", Family Relations, vol. 12, no. 3, (1981), pp. 55-60.

[5] S.A. McLaughlin, D. Doezema and D.P. Sklar, "Human Simulation in Emergency Medicine Training: A Model Curriculum", Academic Emergency Medicine, vol. 9, no. 11, (2008), pp.1310-1318.

[6] Ministry of Gender Equality \& Family, Youth Harmful Environmental Survey, (2012). 
[7] Y. Sung, D. Kim, H.J. Park and K.M. Park, "Bayesian Probability-based UI Control Framework of Sexual Violence Prevention Education Apps. Workshop on Multimedia 2015", Jeju National University International Center, Jeju Island, South Korea, (2015).

[8] J.O. Berger, "Statistical Decision Theory and Bayesian Analysis. Springer Series in Statistics", Springer-Verlag, (1985).

[9] P.Y. Kim, J.W. Kim and Y. Sung, "Bayesian Probability-based Hand Property Control Method", International Conference on Intelligent Technologies and Engineering Systems, Kaohsiung, Taiwan, (2014).

[10] J. Son and Y. Sung, "Bayesian Probability and User Experience-based Smart UI Design Method", International Conference on Intelligent Technologies and Engineering Systems, Kaohsiung, Taiwan, (2014).

\section{Authors}

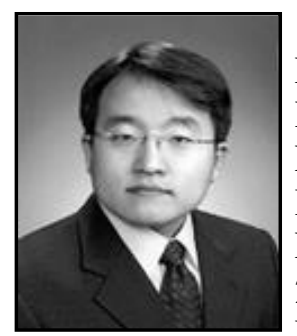

Yunsick Sung, he is currently an Assistant Professor in the Falculty of Computer Engineering at Keimyung University, Daegu, Republic of Korea. He received the BS degree in Division of Electrical and Computer Engineering from Pusan National University, Busan, Republic of Korea, in 2004, the MS degree in Computer Engineering from Dongguk University, Seoul, Republic of Korea, in 2006, and the Ph.D degree in Game Engineering from Dongguk University, Seoul, Republic of Korea, in 2012. He was employed as a Member of the Researcher at Samsung Electronics in Republic of Korea, between 2006 and 2009. He was the Plural Professor at Shinheung College, Gyeonggi-do, Republic of Korea, in 2009, and at Dongguk University, Seoul, Republic of Korea, in 2010. He was also the postdoctoral fellow at University of Florida, Florida, USA, between 2012 and 2013. His research interests are focused on the areas of Games, Pervasive Computing, and Robotics.

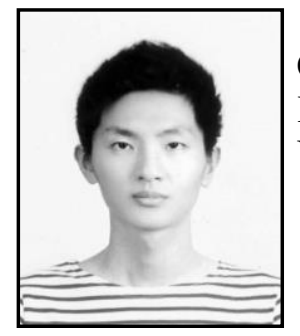

Donguk Kim, he is currently an undergraduate in the Falculty of Computer Engineering at Keimyung University, Daegu, Republic of Korea. His research interests are focused on the areas of Games and UAVs.

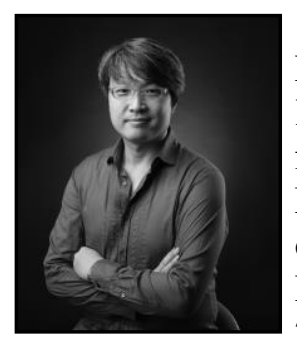

Hyung-Jin Park, he is currently an Associate Professor in the Faculty of Art \& Media at Keimyung University, Daegu, Republic of Korea. He received the B.A. degree in Division of Product Design from Hong-Ik University, Seoul, Republic of Korea, in 1998, the M.A. degree in Animation from Hong-Ik University, Seoul, Republic of Korea, in 2004, and he completed doctoral course in Film \& Digital Media from Hong-Ik University, Seoul, Republic of Korea, in 2011. He was employed at Shin-Il Inc. in Republic of Korea, between 1998 and 2000. He had a number of TV animation the planning and production, between 2000 and 2004. He was the Adjunct Professor at Daegu University, Daegu, Republic of Korea, between 2002 and 2004, and a part-time lecturer at Hong-Ik University, Seoul, Republic of Korea, in 2004. 


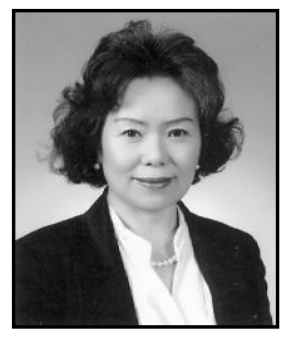

Kyung Min Park, she is currently a Professor in the Major of Community Health Nursing, the department of Nursing, College of Nursing, Keimyung University, Daegu, Republic of Korea. She received the BS degree in Division of Home Economics from Korea National Open University, Seoul, Republic of Korea, in 1985, the MPH degree in Public Health from KyungPook National University, Daegu, Republic of Korea, in 1987, and the Dr.PH degree in Public Health from KyungPook National University, Daegu, Republic of Korea, in 1992. She was the postdoctoral fellow at Johns Hopkins University at Baltimore, USA, between 1993 and 1994. She was an Assistant Professor and Associate Professor in the Faculty of Department of Health Administration at Sunlin College, Pohang, Republic of Korea, between 1988 and 1996. Her research interests are focused on the areas of Simulation, PBL and Community Health Nursing. 